\title{
Impaired oesophageal transit of capsule versus tablet formulations in the elderly
}

\author{
A C Perkins, C G Wilson, P E Blackshaw, R M Vincent, R J Dansereau, K D Juhlin, \\ P J Bekker, R C Spiller
}

\begin{abstract}
Drug induced oesophageal injury is an important and preventable cause of iatrogenic injury. In most cases the injury is considered to be due to mucosal contact from formulations lodged in the oesophagus. A scintigraphic study was performed comparing the oesophageal transit of enteric coated tablets with similar sized and shaped gelatin capsules, using a population of elderly healthy volunteers similar in age (50-79 years) to the population most likely to be receiving regular treatment. Twenty three volunteers injested the radiolabelled tablet or capsule with $50 \mathrm{ml}$ of water while sitting on two separate occasions according to a randomisation schedule. Oesophageal transit was assessed by gamma scintigraphy. Gastric residence was also assessed in 11 of 23 subjects. While the tablet was readily cleared from the oesophagus, mean transit time $4 \cdot 3$ seconds (range $1 \cdot 0-14 \cdot 0$ ), the capsule often showed a comparatively prolonged holdup, mean transit time 20.9 seconds (range $1 \cdot 5-174 \cdot 5$ ). Ten of 11 tablets emptied from the stomach intact, while all 11 capsules broke up in the stomach. Gelatin capsules showed a clear tendency to remain within the oesophagus of healthy elderly volunteers, while similar sized enteric coated tablets did not. These studies show the importance of assessing oesophageal transit when designing the formulation of drugs with a potential for oesophageal injury.

(Gut 1994; 35: 1363-1367)
\end{abstract}

Medical Physics

A C Perkins

P E Blackshaw

R M Vincent

and Therapeutics,

University Hospital,

Nottingham

R C Spiller

Department of

Pharmaceutical

Sciences, University of

Strathclyde, Glasgow

$\mathrm{C}$ G Wilson

Procter and Gamble Pharmaceuticals, Norwich, USA

R J Dansereau

K D Juhlin

P J Bekker

Correspondence to:
Dr A C Perkins, Department

of Medical Physics,

of Medical Physics,

Nottingham NG7 2UH.

Accepted for publication

17 January 1994

Although painful dysphagia resulting from drug induced oesophagitis is comparatively uncommon, ${ }^{1}$ such cases probably reflect the tip of the iceberg. When endoscopic surveillance has been routinely performed in healthy volunteer studies, oesophagitis is detectable in $20 \%$ of subjects taking certain non-steroidal anti-inflammatory drugs (NSAIDs). ${ }^{2}$ This mucosal injury is seen with a wide range of substances including most commonly tetracycline, emepronium bromide, slow release potassium chloride, and NSAIDs. Most injuries are seen in the mid-lower oesophagus, this being where other studies ${ }^{3-5}$ have shown that certain formulations tend to lodge. Prolonged mucosal contact of many drugs is toxic through a range of mechanisms including low or high $\mathrm{pH}$ as well as high osmolarity. ${ }^{3} \mathrm{~A}$ number of studies have suggested that gelatin capsules in particular show a tendency to adhere to oesophageal mucosa once they start to take up water. We therefore set out to compare the oesophageal transit of two formulations, one an enteric coated tablet and another a similar sized, filled gelatin capsule. As patients receiving regular treatment are usually elderly we chose a group of healthy elderly volunteers to assess the effect of formulation on oesophageal transit.

\section{Methods}

FORMULATIONS

Placebo tablets and capsules were supplied by Procter and Gamble Pharmaceuticals. The dosage forms were enteric coated tablets and \#3 hard gelatin capsules containing enteric coated beads. The tablets were modified oval shaped $(5.7 \times 11.5 \mathrm{~mm})$ weighing $240 \mathrm{mg}$ with a density of $1.2 \mathrm{~g} / \mathrm{cc}$. The enteric coating consisted of a Eudragit L100-55 (Rohm Pharma, Darmstadt, West Germany) polymer system with an average film thickness of 50 microns. The \#3 gelatin capsules contained roughly 400 enteric coated 20/25 mesh Nu-Pareil beads (Crompton \& Knowles, Fairlawn, NJ) coated with the same Eudragit L100-55 polymer system with an average film thickness of 40 microns. The density of the filled capsule is $0.6 \mathrm{~g} / \mathrm{cc}$.

Placebo formulations were prepared because it is the exterior of the dosage form that affects adhesion and elimination of the drug simplified the execution of the study.

\section{RADIOLABELLING}

The units were radiolabelled in a clean 


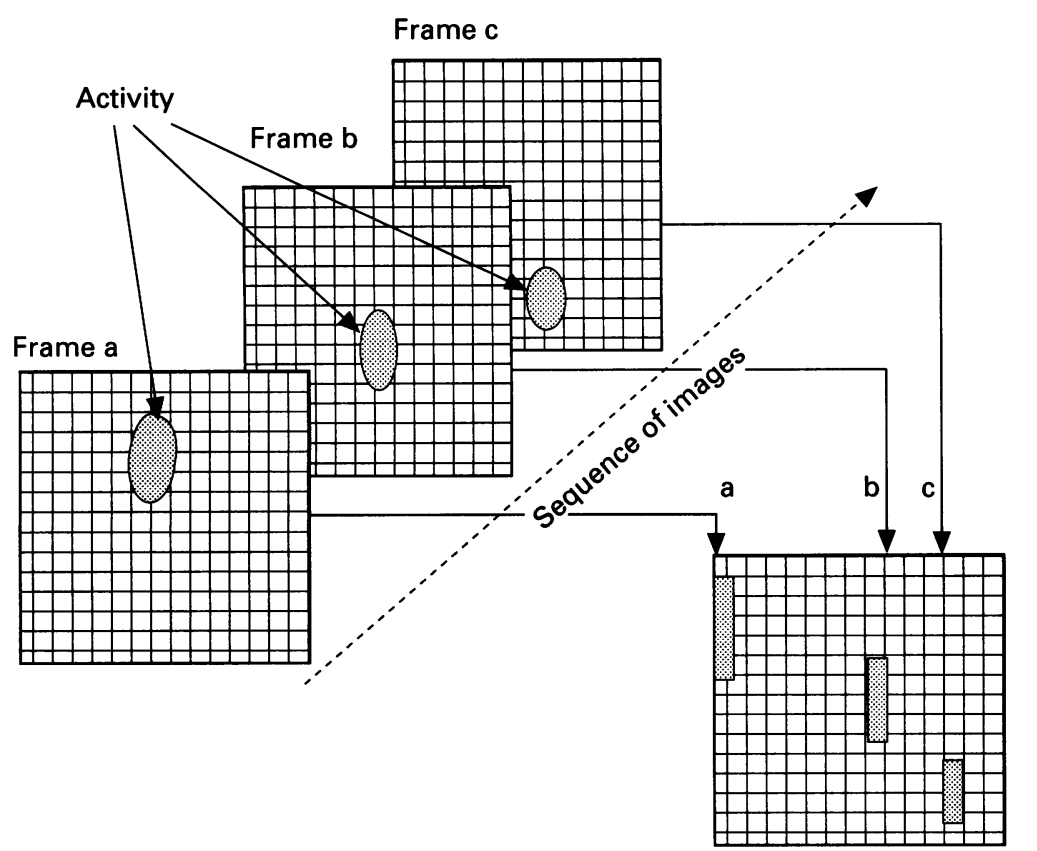

Condensed image display

Figure 1: The condensed image is generated from each frame in the sequence of images. By summing all the counts in the $x$ direction in the image matrix and putting the result in one pixel with the $x$ coordinate corresponding to the frame number the condensed image may be generated. Each column therefore represents one frame in the study, the final image displaying the vertical position of the activity ( $y$ axis) against time ( $x$ axis).

exchange resin IRA $416(\mathrm{Cl})$, particle size $0.3-1 \cdot 2 \mathrm{~mm}$ (BDH Laboratories Lot $0293140 \mathrm{~K})$. The mixture was then dried in a glass beaker using a hot air dryer.

\section{Tablets}

The tablets were clamped and drilled at one edge using a $1.5 \mathrm{~mm}$ diameter drill bit sterilised with alcohol. Radiolabelled resin was tamped into the interior of the tablet using a modified Eppindorf pipette tip to facilitate loading of the resin in the fine drill hole. The units were finally sealed with a small amount of epoxy resin glue. The tablets were visually inspected to ensure that the glued area was smooth and there were no cracks in the film resulting from the drilling process.

\section{Capsules}

Capsules were placed upright in a perspex support and opened. The cap was placed on a cléan tissue: Using a microspatula, about $5 \mathrm{mg}$ of the contents was removed. Radiolabelling was performed by the addition of $5 \mathrm{mg}{ }^{99 \mathrm{~m}} \mathrm{Tc}$ labelled amberlite resin. The shell was firmly capped and the capsule inverted six times to mix the contents. In each case units were weighed before and after radiolabelling.

The amount of radioactivity incorporated into the formulations was assayed immediately after radiolabelling the units using a Vinten Isocal, ionisation chamber dose calibrator (Type NO 282/L/1, serial number 88231). The activity at the time of administration was then calculated, accounting for the time delay between radiolabelling and administration. The radioactive doses were again calibrated in the dose calibrator immediately before dosing the volunteers.

\section{STUDY POPULATION}

Twenty three volunteers aged 50 years or over were recruited from the surrounding population of Nottingham. The mean age of the subjects was 66 years (range 50-79). Table I gives the details of volunteers. During the two weeks before the trial and immediately after completion of the study all subjects had a medical examination. Subjects were divided into four groups and dosed according to a random schedule. A minimum period of three days was permitted between each investigation.

The study was approved by the hospital ethical committee and the Administration of Radioactive Substances Advisory Committee (ARSAC) of the UK Department of Health. All subjects were fully informed of the procedure and gave written consent to participate.

\section{STUDY PROCEDURE}

The volunteers arrived on each of the study mornings having fasted from 2200 the previous evening. At 0800 each subject was given a light breakfast of two slices of buttered toast and tea with milk. In strict order commencing at 1100 the subjects were seated in front of the gammacamera to facilitate anterior imaging. In each case the subject received the dosage form with $50 \mathrm{ml}$ water taken in a continuous sequence of swallows. The images were recorded in a $64 \times 64$ cell matrix, over a total imaging time of 10 minutes as follows: (a) 60 dynamic frames ( 2 frames per second), (b) 38 dynamic frames (1 frame per 15 seconds).

Gastric emptying of the dosage forms was assessed in the first 11 consecutive volunteers. External radioactive markers were then taped to the anterior and posterior abdomen to permit accurate alignment of the subsequent sequential static images. Anterior and posterior

TABLE I Details of subjects included in the oesophageal transit study

\begin{tabular}{|c|c|c|c|}
\hline Subject & Age (y) & Sex & Dosing schedule \\
\hline 1 & 71 & $\mathbf{M}$ & A \\
\hline 2 & 74 & $\mathrm{~F}$ & $\mathbf{A}$ \\
\hline 3 & 71 & $\mathbf{M}$ & A \\
\hline 4 & 70 & $\mathrm{~F}$ & B \\
\hline 5 & 69 & $\mathrm{~F}$ & B \\
\hline 6 & 71 & $\mathbf{M}$ & B \\
\hline 7 & 64 & $\mathrm{~F}$ & A \\
\hline 8 & 69 & $\mathrm{~F}$ & B \\
\hline 9 & 65 & $\mathbf{M}$ & B \\
\hline 10 & 71 & $\mathbf{M}$ & A \\
\hline 11 & 50 & $\mathrm{~F}$ & B \\
\hline 12 & 64 & $\mathbf{M}$ & $\overline{\mathbf{A}}$ \\
\hline 13 & 65 & $\mathbf{F}$ & B \\
\hline 14 & 70 & $\mathbf{M}$ & B \\
\hline 15 & 55 & $\mathrm{~F}$ & B \\
\hline 16 & 58 & $F$ & B \\
\hline 17 & 67 & $\mathbf{F}$ & $\bar{A}$ \\
\hline 18 & 64 & $\mathbf{M}$ & A \\
\hline 19 & 64 & $\mathrm{~F}$ & A \\
\hline 20 & 69 & $\mathbf{M}$ & B \\
\hline 21 & 58 & $\mathbf{F}$ & B \\
\hline 22 & 79 & $\mathbf{M}$ & A \\
\hline 23 & 56 & $\mathbf{F}$ & B \\
\hline
\end{tabular}

$\mathrm{A}=$ Capsule followed by tablet; $\mathrm{B}=$ tablet followed by capsule. 
TABLE II Transit times (sec) for tablets and capsules

\begin{tabular}{lcc}
\hline Subject & Capsule & Tablet \\
\hline 1 & $7 \cdot 50$ & $14 \cdot 00$ \\
2 & $9 \cdot 00$ & $6 \cdot 00$ \\
3 & $3 \cdot 00$ & $3 \cdot 00$ \\
4 & $4 \cdot 50$ & $3 \cdot 00$ \\
5 & $6 \cdot 50$ & $3 \cdot 50$ \\
6 & $16 \cdot 00$ & $4 \cdot 00$ \\
7 & $14 \cdot 50$ & $2 \cdot 00$ \\
8 & $3 \cdot 50$ & $4 \cdot 00$ \\
9 & $4 \cdot 50$ & $1 \cdot 50$ \\
10 & $36 \cdot 00$ & $2 \cdot 50$ \\
11 & $17 \cdot 50$ & $6 \cdot 00$ \\
12 & $5 \cdot 00$ & $8 \cdot 00$ \\
13 & $35 \cdot 00$ & $2 \cdot 00$ \\
14 & $5 \cdot 00$ & $4 \cdot 00$ \\
15 & $9 \cdot 50$ & $2 \cdot 50$ \\
16 & $38 \cdot 50$ & $6 \cdot 50$ \\
17 & $1 \cdot 50$ & $1 \cdot 00$ \\
18 & $15 \cdot 50$ & $4 \cdot 00$ \\
19 & $174 \cdot 50$ & $2 \cdot 50$ \\
20 & $7 \cdot 50$ & $2 \cdot 50$ \\
21 & $11 \cdot 00$ & $8 \cdot 00$ \\
22 & $15 \cdot 00$ & $2 \cdot 00$ \\
23 & $40 \cdot 00$ & $7 \cdot 00$ \\
Mean & $20 \cdot 90$ & $4 \cdot 33$ \\
Sample SD & $35 \cdot 60$ & $2 \cdot 95$ \\
Range & $1 \cdot 5-174 \cdot 5$ & $1 \cdot 0-14 \cdot 0$ \\
Population SD & $34 \cdot 80$ & $2 \cdot 88$ \\
& & \\
\hline
\end{tabular}

images (each of 30 second duration) of the stomach were recorded every 20 minutes until gastric emptying was completed.

\section{PROCESSING AND DISPLAY OF SCINTIGRAPHIC} DATA

All images were analysed by computer (Gamma 11, Nuclear Diagnostics Ltd London/Stockholm) by one of two experienced operators (RMV and ACP), who were blind to the dosing schedule and working to the same previously defined criteria. In each case the individual frames in the study were first displayed to determine the time that activity was first seen on the oropharynx. The subsequent frames were displayed to show the time of arrival of the preparation in the stomach. The difference in time between these frames was used to give the oesophageal transit time of the formulation in seconds. Once the initial transit time was recorded, the software application was used to produce the condensed image display. Dynamic frames were analysed by defining regions of interest over the oropharynx, oesophagus, and stomach. A condensed image format displaying transit over the first 30 seconds and the first 10 minutes was produced. The display is produced by adding the collected data across the $40 \mathrm{~cm}$ field of view of the detector to form a single column of data for each frame. By

TABLE III Gastric residence times and site of disintegration for tablets and capsules

\begin{tabular}{llll}
\hline Capsule & & & Tablet \\
\cline { 1 - 1 }$t_{1 / 2}$ (min) & $\begin{array}{l}\text { Site of } \\
\text { break up }\end{array}$ & $\begin{array}{l}\text { Time to } \\
\text { empty (min) }\end{array}$ & $\begin{array}{l}\text { Site of } \\
\text { break up }\end{array}$ \\
\hline 26 & Stomach & 33 & Small intestine \\
24 & Stomach & 37 & Small intestine \\
47 & Stomach & 32 & Small intestine \\
51 & Stomach & 28 & Small intestine \\
24 & Stomach & 78 & Small intestine \\
43 & Stomach & 32 & Small intestine \\
45 & Stomach & 96 & Small intestine \\
22 & Stomach & 11 & Small intestine \\
37 & Stomach & 44 & Small intestine \\
23 & Stomach & 11 & Small intestine \\
23 & Stomach & $\left(\mathrm{t}_{1 / 2}\right) 26$ & Stomach 12 min \\
\hline
\end{tabular}

TABLE IV Classification of subjects according to the results of transit of the two formulations

\begin{tabular}{lllc}
\hline & \multicolumn{3}{c}{$\begin{array}{l}\text { Oesophageal dysmotility with } \\
\text { the tablet formulation }\end{array}$} \\
\hline & & Yes & No \\
Oesophageal dysmotility with & Yes & 0 & 5 \\
the capsule formulation & No & 0 & 18 \\
\hline
\end{tabular}

placing all columns of data in sequence the condensed image is produced. Figure 1 gives a schematic diagram of the production of the condensed images.

Anterior and posterior static images of the gastric activity were displayed and emptying was assessed by experienced operators (PEB and ACP) after defining a region of interest over the stomach and recording counts from the images. All data were corrected for radionuclide decay, background subtracted, and a geometric mean value calculated according to the method of Hardy and Perkins. ${ }^{7}$ The time of break up of the formulation was noted, together with the time at which the unit or greater than $50 \%$ of the unit left the stomach.

\section{Results}

A total of 46 oesophageal transit studies were carried out. No adverse reactions or events were reported. All the volunteers swallowed the units with the $50 \mathrm{ml}$ of water provided. There were no requests for additional water. None of the subjects complained of any discomfort or problem in swallowing any of the units. Volunteers were not aware of the data at any time. The subjects were therefore ignorant of any dysmotility that occurred.

Tables II and III show the results of the transit and site of break up of the formulations. Of the 23 tablet studies the mean transit time was 4.3 seconds with a range of 1.0 to 14 seconds (sample standard deviation 2.9 seconds). Of the 23 capsule studies the mean transit time was 20.9 seconds with a range of 1.5 to 174.5 (sample standard deviation $35 \cdot 6$ ). A statistical analysis was conducted to quantify the degree of difference between the two formulations. For each formulation, subjects were classified as having oesophageal adhesion or not, depending upon whether the oesophageal transit time was greater than 20 seconds. Table IV gives the final classification of the results for the 23 subjects. The cells of Table IV that provide information on the difference between the formulations are the Yes/No cells. Although McNemar's test is generally used in such situations, the small sample size in this study required the use of a conditional exact binomial test, ${ }^{8}$ in which the null hypothesis specifies the conditional probability associated with each of the two cells as 0.5 . The computed $p$ value was $p=0.0626$, which is a moderate indication of a difference between the transit of the two formulations.

The condensed image display provided a useful visual method for showing the patterns of dysmotility in the subjects (see Figs 2 and 3). The patterns of dysmotility evident from the condensed images fell into two types. 

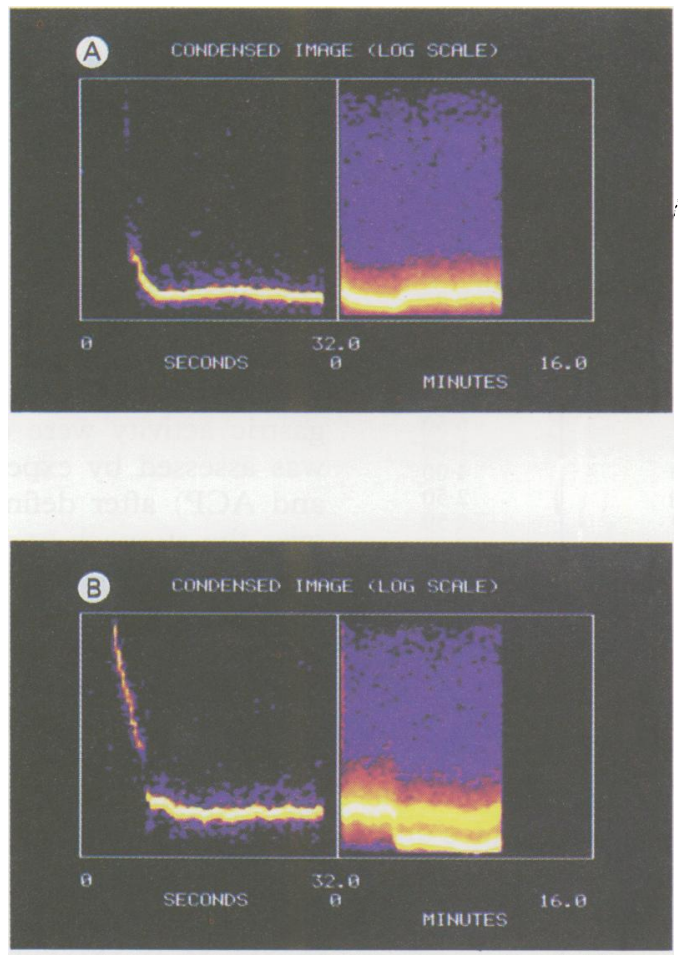

Figure 2: Examples of the functional image display of one subject. The vertical axis represents distance between the oropharynx and the stomach and the horizontal axis represents time. In each case the left hand trace shows the position of the unit over the first 30 seconds and the right hand trace shows the position over the full 10 minute study. The trace therefore shows the position of the unit with time. Normal transit of both the tablet $(A)$ and capsule $(B)$ can be seen as all the activity promptly arrives at the stomach.

Oscillatory motion (seven studies) - oscillation in the upper-mid oesophagus was visualised in four studies and oscillatory motion in the lower oesophagus was seen in three studies.

Oesophageal stasis (18 studies) - stasis was seen in the upper oesophagus in three studies, the mid oesophagus in nine studies, and the lower oesophagus in six studies.

From the gastric emptying data given in Table III it can be seen that one of the 11 tablet preparations broke up in the stomach before emptying. The gastric images of the 11 subjects when receiving the capsule preparations showed that the capsule broke up within the stomach in every case. The mean emptying half time $(50 \%$ or greater) for the tablet preparation was 19 minutes (range 11-96). The $50 \%$ emptying time for the capsule preparations was 33 minutes (range 22-51).

\section{Discussion}

Radiographic studies and endoscopy have previously been used to examine the pathology of oesophageal injuries. Previous fluoroscopic studies $^{9}$ have been performed to assess the oesophageal transit of tablets and capsules given orally but, in such instances although the shape and size of the preparation was unaltered for this study, the addition of barium sulphate granulate affected the density of the formulation. Such a change in density would therefore affect the alimentary transit of the
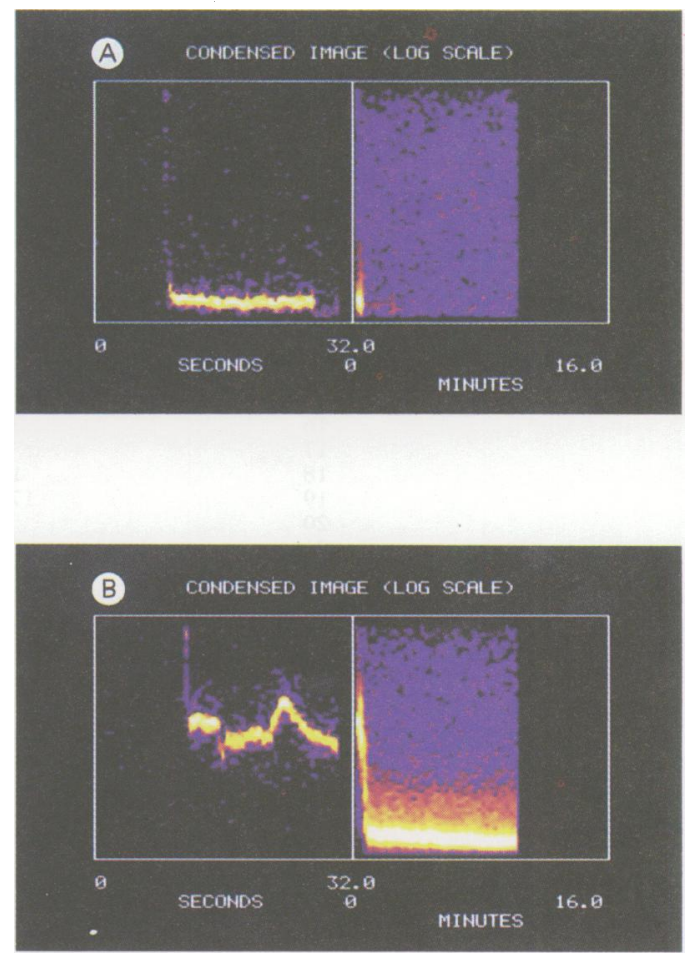

Figure 3: Example of the functional image display from one subject, showing normal tablet transit $(A)$ with delayed transit of capsule (B). The condensed image showing the transit of the capsule over the first 30 seconds shows retention of activity in the mid oesophagus with an episode of oesophageal spasm at between 20 and 25 seconds after the start of the swallow.

units. Scintigraphic studies of the oesophageal transit of liquid and solids provide a more physiological means of assessing oesophageal transit and can show oesophageal spasm, stasis, and gastric reflux. ${ }^{10-13}$ Oesophageal transit is a routine clinical procedure in nuclear medicine departments, the method being noninvasive and with minimal discomfort to patients. A logical extension of this diagnostic procedure is to assess the transit of radiolabelled tablets and capsules and for all such studies it is essential that the radiolabelling does not adversely affect the contact surface properties of the preparation. Similar methods have been used to examine the influence of posture and coadministration of water. ${ }^{14}$

The results show some unexpected findings concerning the oesophageal transit of the formulations studied. Oesophageal stasis with complete dissolution of preparation has been previously seen in some studies but this phenomenon did not occur in this study. The transit seen suggested a degree of oesophageal dysfunction with some of the capsule preparations, though volunteers were initially screened and those with clinically apparent oesophageal dysmotility were excluded. The condensed image display was especially helpful in evaluating the transit of the preparations in each case. It is significant that the capsule was slow to clear in $22 \%$ of the studies, according to the mutually agreed preset criterion that oesophageal transit times of 20 seconds or greater would be taken as an indication of 
adhesion. The 20 second limit is less, however, than that used in other published studies ${ }^{15} 16$ where 60 and 90 seconds have been taken as the upper limit. In our experience, using scintigraphic studies, normal swallowing occurs within this shorter time period. Irrespective of the cut off used, the clear difference in the transit times measured between the tablets and capsules in this study remains apparent.

The tablets and capsules used in this study were of similar size and shape (the tablets being ovoid), however, as the capsules were only half the density of the tablets this may in part account for the differences seen in the comparative transit times of the two formulations. It is not possible, therefore, to conclude from this study whether the slower transit of the capsules was caused by oesophageal adhesion, or merely lack of propulsion because of the lower density of the units. The method of dosing is also extremely important. Drinking the water in a continuous sequence of swallows or alternatively by pausing between swallows may lead to different peristaltic contractions and hence a different pattern of transit. This study was performed in a standard manner with subjects swallowing in a continuous sequence of swallows.

The site of dysmotility seen in this study was mainly in the mid to lower oesophagus, this being consistent with other reported studies of oesophageal transit. ${ }^{5}$ Failure of propulsion in the distal oesophagus has also been noted in patients with non-specific motility disorders. The peristaltic wave tends to travel part of the way down the oesophagus and then to fail to progress, the most common site of failure being just below the aortic arch. ${ }^{17}$ Peristaltic dysfunction with hypotensive peristalsis in the distal oesophagus has also been seen in patients with overt reflux. ${ }^{18}$ The prolonged residence of the capsules in the distal oesophagus is probably related to minor subclinical impairment of propulsion as well as the mucous adhesion properties of the capsule surface. It may be that the 'stickiness' of the gelatin capsule is related to the moisture content in the saliva, thus accounting for the differences between normal subjects.

In addition to the impaired propulsion seen with the capsules it is important to consider the consequences of the break up of the formulation within the stomach, a feature that was seen in all 11 subjects on swallowing the capsules and only one subject swallowing the tablet. The significance of this finding is uncertain because the method of manufacture may affect the disintegration characteristics of the units. In the case of the capsules the radiolabelling and manufacturing procedure should not affect the disintegration characteristics, but this may not be the case for the tablets as these had been drilled and plugged. In one subject the capsule was seen to break up before entering the stomach. This was seen on the condensed image as a double diverging trace. Emptying of the contents of the capsule into the stomach would have the secondary problem of oesophageal injury should gastrooesophageal reflux occur. Although gastrooesophageal reflux was not seen in this study, the occurrence of this cannot be ignored in the design of formulations intended for elderly patients.

This study clearly shows the importance of the assessment of oesophageal transit of formulations of drugs with a potential for oesophageal injury.

The authors would like to thank Vikram Patel, Procter and Gamble Pharmaceuticals for advice with the design of the study and Dr Malcolm Frier, Principal Radiopharmacist, University Hospital Nottingham, for assistance with manufacturing and radiolabelling.

1 Kikendall JW, Friedman AC, Oyewole MA, Fleischer D, Johnson LF. Pill induced oesophageal injury: case report and a review of medical literature. Dig Dis Sci 1983; 28: 174-82.

2 Santucci L, Patoia L, Fiorucci S, Farroni F, Favero D, Morelli A. Oesophageal lesions during treatment with piroxicam. BMF 1990; 300: 1018.

3 Pemberton J. Oesophageal obstruction and ulceration caused by oral potassium therapy. Br Heart $\mathcal{F} 1970$; 32: caused

4 Evans KT, Roberts GM. Where do all the tablets go? Lancet 1976; ii: 1237-9.

5 Eng J, Sabanathan S. Drug induced oesophagitis. Am $\mathcal{f}$ Gastroenterol 1991; 86: 1127-33.

6 Wilson CG, Hardy JG. Gastrointestinal transit of an osmotic tablet drug delivery system. F Pharm Pharmacol 1985; 37: 573-5.

7 Hardy JG, Perkins AC. Validity of the geometric mean correction in the quantification of whole bowel transit. Nucl Med Commun 1985; 6: 217-24.

8 Lehmann EL. Testing statistical hypotheses. New York: John Wiley, 1959.

9 Channer KS, Virjee JP. The effect of size and shape of tablets on their oesophageal transit. $\mathcal{f}$ Clin Pharmacol 1986; $26: 141-6$.

10 Ham HR, Geoges B, Guillaume M, Erbsmann F, Dobbelier. Evaluation of methods for qualitative and quantitative assessment of oesophageal transit of liquid. Eur f Nucl Med 1985; 11: 17-21.

11 Klein HA, Wald A. Computer analysis of radionuclide oesophageal transit studies. $f \mathrm{Fucl}$ Med 1984; 25: 957-64.

12 Svedberg JB. The bolus transport diagram: a functional display method applied to oesophageal studies. Clin Phys Physiol Meas 1984; 3: 267-72.

13 Tolin RD, Malmud LS, Reilley J, Fisher RS. Oesophageal scintigraphy to quantitate oesophageal transit.

14 Fisher RS, Malmud LS, Applegate G, Rock E, Lorber SH Effect of bolus composition on esophageal transit: concise Effect of bolus composition on esophageal transit:

15 Hey H, Jorgensen F, Sorensen K, Hasselbalch H, Wamberg T. Oesophageal transit of six commonly used tablets and capsules. BMF 1982; 285: 1717-9.

16 Jorgensen F, Hesse B, Tromholt N, Hojgaard L, Stubgaard M. Esophageal scintigraphy: reproducibility and normal ranges. $\Im$ Nucl Med 1992; 33: 2106-9.

17 Kahrilas PJ, Dodds WJ, Kern M, Arndorfer RC, Reece A. Oesophageal peristaltic dysfunction in peptic oesophagitis. Gastroenterology 1986; 91: 897-904.

18 Kahrilas PJ, Dodds WJ, Hogan WJ. Effect of peristaltic dysfunction on oesophageal volume clearance. Gastroenterology 1988; 94: 73-80. 